\title{
Approach to probiotics in pediatrics: the role of Lactobacillus rhamnosus GG
}

a. Task Force on Pediatric Gastroenterology and Nutrition, Hospital General de Agudos Dr. Ignacio Pirovano, Autonomous City of Buenos Aires, Argentina.

b. Department of Mother and Child Health, Hospital Universitario Austral, Pilar, Argentina.

c. Outpatient Clinic of Pediatric Gastroenterology and Nutrition, Hospital Pediátrico Federico Falcón, Pilar, Argentina.

d. Department of Pediatrics, Grupo Médico Las Lomas, San Isidro, Argentina.

e. Sanatorio de Niños de Rosario, Rosario, Argentina.

f. Institute of Industrial Dairy Science (CONICET-UNL), School of Chemical Engineering, Universidad Nacional del Litoral, Santa Fe Argentina.

E-mail address:

Gabriel Vinderola: gvinde@fiq.unl.edu.ar

Funding:

None.

Conflict of interest: Mead Johnson Nutrition Argentina facilitated the meetings and technical details of this study. The manuscript was written with the help of all authors in an autonomous manner; the company did not interfere with the editorial management or the final article. The authors state that their only relationship with Mead Johnson Nutrition Argentina was their participation in conferences and symposiums organized by the company, as in others carried out by other companies.

Received: $12-22-2020$ Accepted: 8-4-2021

\author{
Christian Boggio Marzet ${ }^{a}$ (D), Fernando Burgos ${ }^{b}$ (D), Mónica Del Compare ${ }^{c}$ (D), \\ Ingrid Gerold ${ }^{(\mathbb{D}}$, Omar Tabacco ${ }^{\text {(D) }}$, Gabriel Vinderolaf $(\mathbb{D})$
}

\begin{abstract}
Preterm birth, C-sections, antibiotics, and limited breastfeeding contribute to the increase in noncommunicable diseases. Our objective was to perform a descriptive review of probiotic use in pediatrics, focused on Lactobacillus rhamnosus GG. Certain probiotics have demonstrated to be effective in acute diarrhea and antibiotic-associated diarrhea. $L$. rhamnosus GG and Saccharomyces boulardii may shorten their duration and symptoms. L. reuteri DSM 17938 and L. rhamnosus GG were effective to manage infant colic. The use of this strain in infant formulas for cow's milk protein allergy may promote an earlier tolerance acquisition. In relation to the prevention of atopic dermatitis, the administration of L. rhamnosus GG during pregnancy reduced its development in the infant. The use of probiotics as adjuvants is a possibility to consider in current pediatric practice.

Key words: microbiota, probiotics, infant formulas, Lactobacillus rhamnosus GG.
\end{abstract}

http: / / dx.doi.org/10.5546/ aap.2022.eng.e1

To cite: Boggio Marzet, Burgos F, Del Compare M, Gerold I, et al. Approach to probiotics in pediatrics: the role of Lactobacillus rhamnosus GG. Arch Argent Pediatr 2022;120(1):e1-e7.

\section{INTRODUCTION}

The combination of factors like preterm birth and low birth weight, elective C-sections, antibiotic use in the first 2 years of life, and limited breastfeeding contributes to an increase in noncommunicable diseases among children. ${ }^{1,2}$ Early microbial interventions, such as those including probiotics, may be a complementary nutritional resource for the management of some of these conditions. ${ }^{3}$ The rationale for this study is to offer an update on the potential benefit of probiotic cultures for the prevention and treatment of certain chronic conditions that may develop in the first years of life. The objective was to perform a descriptive review of probiotic use in pediatrics, focused on Lactobacillus rhamnosus GG (LGG).

This article falls under the category "special articles", being a descriptive review of the subjet. Each author conducted a review of studies published in Pubmed selecting those they considered relevant for inclusion in this article. CBM focused on the aspects related to allergies; FB centered on the aspects related to the mechanisms of action; MDC analyzed safety-related issues; IG described the benefits; OT explained perinatal colonization and probiotic use in neonatology; and GV described microbiological aspects. Key words included in the bibliographic search were probiotics, microbiome, allergy, safety, perinatal colonization, infants, mechanisms of action, and health benefits.

\section{PERINATAL INTESTINAL COLONIZATION}

The view we have of our relationship with microorganisms 
has changed from being considered exclusively pathogens, to being fundamental members of a "healthy microbial environment" for the development of children and their immune, metabolic, and neurological maturation, ${ }^{4,5}$ since birth.

Perinatal colonization is influenced by the microbiome of the female reproductive system, where the Lactobacillus genus prevails. ${ }^{2}$

The uterine environment is not sterile either. The presence of microbes in the placenta, amniotic fluid, and meconium has been demonstrated, ${ }^{6}$ but it is not possible yet to speak of a "fetal microbiome".

The moment of birth and the first hours of life define the infant's microbiome. ${ }^{7}$ There are taxonomic differences between the microbiome of neonates born via vaginal delivery and via C-section. Breastfeeding, ideally initiated in the first hour after birth, provides microorganisms, specific oligosaccharides, and immune factors. ${ }^{8}$ The functional effects of breastfeeding, although not strictly nutritional, are related to immunity, maturation, and metabolism, and the infant's microbiome serves as an intermediary.

The mother's diet affects microbial vertical transmission. ${ }^{9}$ Lactobacilli and bifidobacteria are well-known microbial groups due to their benefits in infants in this stage, who are capable of fermenting human milk oligosaccharides and generating an acid setting in the intestinal lumen that hurdles pathogen colonization; in addition, they function as mediators of children's development. ${ }^{1}$

When the factors that promote an adequate perinatal intestinal colonization (term birth, vaginal delivery, breastfeeding) are affected by events such as preterm birth or a C-section or antibiotic use, a microbial intervention with probiotics is an alternative to address the problems resulting from such events.

\section{PROBIOTICS}

In 2002, the World Health Organization established that probiotics are "live microorganisms which when administered in adequate amounts confer a health benefit on the host".${ }^{10}$ Probiotics may be available as dietary supplements or in fermented (yogurts) and not fermented products (infant formulas), ${ }^{11}$ in which viability may be verified. ${ }^{12}$

Probiotics should have a microbiological identification (genus, species, and strain); be safe in the subjects in whom they will be administered; be viable at the time of administration; and have clinical studies that demonstrate their benefits. ${ }^{13}$ Effects are strain-dependent. For example, L. rhamnosus CRL1505 and L. rhamnosus GG are different strains. The former is used in a social education program and is effective in the prevention of airway infections. ${ }^{14}$ The highest number of studies ${ }^{16}$ have focused on the clinical effectiveness of L. rhamnosus GG, isolated by Goldin and Gorbach; ${ }^{15}$ the first human trial was registered in 1995 for the management of acute rotavirus gastroenteritis. ${ }^{17}$

In several cases, the benefits of probiotics are observed without significant modifications in the composition or function of gut microbiota due to the multiple mechanisms of actions involved in the benefits they provide. ${ }^{18}$

\section{PROBIOTICS SAFETY}

The assessment of a strain's safety is a mandatory stage, prior to the determination of probiotic properties, and considers its microbiological identity, route of administration, dosage, and health status of the target population group. ${ }^{19}$

In general terms, lactobacilli are safe and have been included in the positive list of qualified presumption of safety (QPS) established by the European Food Safety Authority. ${ }^{20}$ The risk for infection from these microorganisms was estimated in approximately 1 per every 10 million people. ${ }^{21}$ Several studies have demonstrated the safety of LGG, even in immunocompromised patients (preterm infants, patients with inflammatory bowel disease or human immunodeficiency virus, or older adults, among others). ${ }^{22}$

Patients with severe immunocompromise (acute pancreatitis, major gastrointestinal surgery) may benefit from probiotic use because they are susceptible to sepsis, systemic inflammatory response or multiple organ failure. However, the bibliographic report of certain adverse effects in these patients ${ }^{23}$ makes it necessary to prove the safety of each particular strain in the target condition. Probiotics are not recommended in patients with central venous line, short bowel syndrome or heart valve disease. ${ }^{23}$

Due to the possibility of antibiotic resistance transfer from orally administered strains to microorganisms living in the gut microbiota, molecular technology is used to warrant the absence of antibiotic resistance transfer genes in strains being considered as candidates for 
probiotic use. $^{24}$

L. rhamnosus $\mathrm{GG}^{\circledR}$ is considered safe by the United States Food and Drug Administration (FDA). In Europe, L. rhamnosus strains have a QPS status and their use as infant formula ingredient has been authorized. All L. rhamnosus strains, including LGG, are sensitive to most clinical antibiotics, but resistant to vancomycin; however, this is an inherent factor that is not transferred. ${ }^{25}$

In populations with egg or cow's milk protein allergy, the risk for an allergic reaction does not depend on the probiotic itself, but on the excipients used in its formulation. Certain products marketed as including probiotics showed traces of allergens that were not described in the label. ${ }^{26}$ The European Society for Paediatric Gastroenterology, Hepatology, and Nutrition (ESPGHAN) Committee on Nutrition and the ESPGHAN Working Group for Probiotics and Prebiotics have issued statements about the use of specific strains and the safety aspects to be taken into consideration before their administration. ${ }^{27}$

\section{IMPACT OF PROBIOTICS ON HEALTH}

In recent years, the scientific interest in the benefits of different probiotic strains for the prevention and management of gastrointestinal disorders, metabolic disorders ${ }^{28}$ allergies, ${ }^{29}$ and immune system modulation in general has increased..$^{30}$ In pediatrics, the effectiveness of certain probiotics in the management of acute gastroenteritis, ${ }^{31}$ the prevention and management of antibiotic-associated diarrhea, ${ }^{32}$ infant colic, ${ }^{33}$ and atopic eczema ${ }^{34}$ has been demonstrated.

Certain Saccharomyces boulardii, L. casei, and L. rhamnosus strains have demonstrated their effectiveness in the management of acute diarrhea in children, especially those produced by rotavirus, shortening their duration by 1 day. ${ }^{35,36}$ In relation to antibiotic-associated diarrhea, LGG and S. boulardii are the probiotics with the greatest scientific evidence available. Their administration may reduce the risk for diarrhea by $51 \%$ if started on the same day as antibiotic therapy.

In relation to infant colic, a meta-analysis about the daily administration of L. reuteri DSM 17938 showed a significant reduction in crying time by 51 minutes per day. ${ }^{37}$ LGG also demonstrated to be effective after the administration during 28 days, with a reduction of crying days to a half. ${ }^{38}$

The usefulness of LGG for these gastrointestinal disorders is because of its stability in relation to gastric acidity and bile and can perform its beneficial activity at the intestinal level.

Regarding obesity and metabolic syndrome, it has been demonstrated that the administration of L. plantarum and L. rhamnosus species improved the lipid profile; reduced adipose tissue; reduced total serum cholesterol, LDL cholesterol, and triglycerides; and increased HDL cholesterol. ${ }^{39,40}$

In recent decades, the number of food, respiratory, and skin allergies has increased, which is consistent with the drastic reduction in the gut microbiota diversity and abundance, which, is in turn related to the increase in preterm birth, C-sections, reduced breastfeeding, antibiotic use, lower exposure to the environment, and excessive hygiene. In support of the hygiene theory, epidemiological data have shown a lower incidence of allergies in rural versus urban populations. ${ }^{41}$ In this context, the attention goes to probiotic use for the prevention and management of asthma, atopic eczema, and food allergies. The factors contributing to atopic conditions include alterations in the skin barrier and intestinal mucosa.

The newborn infant's immune system is strongly dominated by $\mathrm{T}$ helper $2\left(\mathrm{~T}_{h} 2\right)$ cells, and the immune response to T helper $1\left(\mathrm{~T}_{h} 1\right)$ cells changes gradually to maintain homeostasis. The $\mathrm{T}_{\mathrm{h}} 2$ phenotype results in higher immunoglobulin $\mathrm{E}$ (IgE) levels and mast cell activation, which in turn increases susceptibility to allergic diseases. The microbiota plays a relevant role in the balance of $T_{h} 1 / T_{h} 2$ immune responses. ${ }^{42}$

The general pathogenesis of an allergic response implies allergen (pollen, food, etc.) sensitization, which induces a transient inflammatory response, with increased allergenspecific IgE levels and migration of effector $\mathrm{T}$ cells, mast cells, and eosinophils to the exposure site. Eosinophils play an important role in respiratory and digestive allergies due to degranulation at the exposure site, which causes immune cell recruitment and tissue damage. Most antigens found in the gastrointestinal tract come from the diet and the gut microbiota and may affect immune tolerance; the antigen presentation of such dietary factors to regulatory T cells is a critical step to prevent an immune response to food antigens. ${ }^{43}$ Any alteration in microbiota levels or diversity may affect mucosal immune tolerance, which leads to food and respiratory allergies. ${ }^{44}$ In addition, low IgA levels in the intestinal barrier may also contribute to 
food allergy. Low IgA levels and the microbiota are related: the gut microbiota may stimulate dendritic cells in Peyer's patches to activate B cells, which causes the production of specific IgA antibodies. Such stimulation may occur through the production of short-chain fatty acids by the microbiota. ${ }^{45}$

The gut microbiota may also affect the development of oral tolerance and sensitization to food antigens. A greater presence of Clostridia and Firmicutes phylum species was related to cow's milk protein allergy in babies. The administration of casein formulas supplemented with LGG to babies with cow's milk protein allergy led to the enrichment of butyrate-producing species, thus promoting tolerance. Supplementation with LGG reduces the risk for other allergic manifestations. ${ }^{46}$

L. rhamnosus GG is one of the most frequently studied probiotics for these conditions. To assess whether atopic dermatitis may be prevented in the first years of life, a study was conducted in 159 pregnant women with a family history of allergy. Participants received LGG $\left(10^{10} \mathrm{CFU}\right)$ or placebo every day for 2-4 weeks before giving birth. After birth, LGG was administered to the mother, who was exclusively breastfeeding, or to the baby for 6 months. The incidence of atopic eczema in the LGG group reduced significantly. ${ }^{34}$ The infants born to mothers who received LGG had a lower risk for atopic dermatitis in their first 2 years of life. ${ }^{47}$

Even with such extent of evidence, further studies are required because a systemic review of 29 clinical studies established that there is not enough evidence to recommend the use of probiotics in the primary prevention of allergic disease, such as asthma or allergic rhinitis. However, the authors suggested their use in pregnant/breastfeeding women with a history of allergy. ${ }^{48}$

\section{PROBIOTICS IN PRETERM INFANTS}

In recent years, the industry of infant formulas has focused its efforts on research and development of the main factors responsible for the functional effects of human milk (oligosaccharides, microorganisms, and metabolites), and the addition of probiotics and prebiotics has demonstrated to be beneficial for the prevention of allergic disease during childhood. ${ }^{16}$

A preterm newborn infant represents an adequate model of vulnerability of the positive factors needed for the development of a healthy gut microbiome due to intestinal immaturity, C-section, antibiotic use, and heterologous feeding; for these reasons, nutritional strategies to improve it have been studied, ${ }^{49,50}$ including probiotics. A systematic review with metaanalysis about probiotic use in preterm infants with a very low birth weight (30 randomized studies and 14 observational studies) showed favorable results across all analyzed variables (lower development of severe enterocolitis, late-onset sepsis, and mortality), although these studies were done using different microorganisms, alone or combined, and diverse dosages. ${ }^{51}$

Another systematic review in 4527 newborn infants born with less than 37 weeks of gestation showed that probiotic use was favorable to reduce the duration of exclusive enteral feeding. That study also showed positive effects on weight gain and postprandial mesenteric blood flow, although different strains and dosages were used, which does not allow to generalize the outcomes to all probiotics. ${ }^{52}$ However, there is consensus about the absence of complications resulting from probiotic use in preterm infants; therefore, such broad margin of safety is a very important aspect to be taken into consideration.

The use of LGG in 640 newborn infants with a very low birth weight (less than $1500 \mathrm{~g}$ ) in the same unit for 8 years showed a higher incidence of severe necrotizing enterocolitis (NEC) after the use of this strain $(10.2 \%$ versus $16.8 \%)$, but no sepsis events were reported. ${ }^{53}$

In a recent publication, the ESPGHAN Working Group for Probiotics and Prebiotics and its Committee on Nutrition established a cautious position about the use of probiotics in preterm infants because they considered that the evidence lacked robustness. In addition, they suggested the need to establish the difference between each probiotic type used, the moment of initiation, dosage, and vehicle (human milk or formula) and emphasized the need to conduct further studies. ${ }^{54}$ On the contrary, another recent report by the American Gastroenterological Association recommends using probiotics in preterm infants born at less than 37 weeks of gestation and with a low birth weight, including LGG among recommended strains, and analyzed parameters such as NEC, overall mortality, late-onset sepsis, and stay in the NICU. ${ }^{55}$

Ultimately, the current evidence is very promising in relation to probiotic use in the field of neonatology in preterm newborn infants, to 
reduce the incidence of main complications, e.g., NEC, late-onset sepsis, and overall mortality, with reliable safety margins. However, the specific probiotic to be used, alone or in combination, the time of treatment initiation and end, and the adequate dosage are yet to be determined. In addition, probiotic use in newborn infants with a very low birth weight (less than $1000 \mathrm{~g}$ ) is still a mystery.

The microbiome of newborn infants is a large community of microorganisms developed in a symbiotic manner and in close relationship with the intestinal immune system. In addition to the potential use of probiotics, general neonatology practice for the management of these patients should be reviewed globally to facilitate the development of healthier bacterial species.

\section{MECHANISM OF ACTION OF PROBIOTICS}

Probiotics may affect the intestinal microorganism by acting on the mucosal immune mechanisms, interacting with commensal or potentially pathogenic microorganisms, generating metabolic products or communicating with the host's cells using chemical signal produced by its metabolism. It has been estimated that these phenomena may explain most of the benefits described for probiotics.

The most relevant mechanisms of action of probiotics include the resistance to pathogen colonization because they may temporarily occupy functional niches left vacant in the residing microbiota, thus preventing opportunistic infections, or may modify the local environment through the production of short-chain fatty acids, lactic acid, bacteriocins, and reactive oxygen species to inhibit pathogen growth. ${ }^{56}$

Certain probiotics contribute to a greater availability of vitamin K, vitamin B12, pyridoxine, biotin, folic acid, nicotinic acid, and thiamin. ${ }^{57}$ Some strains may be considered immunostimulatory due to their ability to induce natural killer cell-mediated immunity. ${ }^{58}$ Others may be considered antiinflammatory due to their ability to induce interleukin- $10 .{ }^{59}$ Probiotics may also activate local macrophages, increasing antigen presentation to B cells and stimulating IgA secretion. ${ }^{60}$

Particularly, among the mechanisms of action described for LGG, it is worth noting that polysaccharides and pili present on its surface allow it to adhere to and temporarily colonize the intestinal mucosa. LGG is able to produce both a biofilm that can mechanically protect the mucosa, and different soluble factors beneficial to the gut by enhancing intestinal crypt survival, diminishing apoptosis of the intestinal epithelium, and preserving cytoskeletal integrity. Moreover, thanks to its lectin-like protein 1 and 2, LGG inhibits some pathogens such as Salmonella. LGG is able to stimulate a non-specific immune response mediated by $\operatorname{IgA}, \operatorname{IgG}$, and $\operatorname{IgM}$ and CD4+ T cell-dependent immune response and, at the same time, promote $T_{h} 1$ immune response by reducing the expression of several activation and inflammation markers on monocytes and increasing the production of interleukin-10, interleukin-12, and tumor necrosis factor- $\alpha$ in macrophages. ${ }^{61}$

\section{CONCLUSIONS}

Preterm birth, low birth weight, elective C-sections, antibiotic use, and limited breastfeeding, among other factors, contributed to the increase in noncommunicable diseases among children. Probiotic use emerges as a safe microbiological strategy in pediatrics for the promotion of intestinal immunity.

L. rhamnosus GG (LGG) and S. boulardii have proven to be safe and effective for the prevention of acute diarrhea and antibioticassociated diarrhea, shortening their duration and reducing their symptoms. In the case of infant colic, both L. reuteri DSM 17938 and LGG can significantly reduce its duration. In relation to the prevention of atopic dermatitis, LGG administered during pregnancy demonstrated to reduce its development in the infant.

Although further evidence is required about the therapeutic and preventive effects of probiotics on food allergies, the studies available to date suggest that the use of LGG in infant formulas for cow's milk protein allergy would promote an earlier tolerance acquisition.

\section{REFERENCES}

1. Milani C, Duranti S, Bottacini F, Casey E, et al. The First Microbial Colonizers of the Human Gut: Composition, Activities, and Health Implications of the Infant Gut Microbiota. Microbiol Mol Biol Rev. 2017; 81:e00036-17.

2. Selma-Royo M, Tarrazó M, García-Mantrana I, GómezGallego C, et al. Shaping Microbiota During the First 1000 Days of Life. Adv Exp Med Biol. 2019; 1125:3-24.

3. Salminen S, Stahl B, Vinderola G, Szajewska H. Infant formula supplemented with biotics: Current knowledge and future perspectives. Nutrients. 2020; 12(7):1952.

4. Douglas-Escobar M, Elliott E, Neu J. Effect of intestinal microbial ecology on the developing brain. JAMA Pediatr. 2013; 167(4):374-9.

5. Sommer F, Bäckhed F. The gut microbiota-masters of host 
development and physiology. Nat Rev Microbiol. 2013; 11(4):227-38.

6. Stinson LF, Boyce MC, Payne MS, Keelan JA. The not-sosterile womb: Evidence that the human fetus is exposed to bacteria prior to birth. Front Microbiol. 2019; 10:1124.

7. Rutayisire E, Huang K, Liu Y, Tao F. The mode of delivery affects the diversity and colonization pattern of the gut microbiota during the first year of infants' life: a systematic review. BMC Gastroenterol. 2016; 16(1):86.

8. Pannaraj PS, Li F, Cerini C, Bender JM, et al. Association between breast milk bacterial communities and establishment and development of the infant gut microbiome. JAMA Pediatr. 2017; 171(7):647-54.

9. Rodríguez JM, Murphy K, Stanton C, Ross RP, et al. The composition of the gut microbiota throughout life, with an emphasis on early life. Microb Ecol Health Dis. 2015; 26:26050.

10. HillC, Guarner F, Reid G, Gibson GR, etal. Expert consensus document: The international scientific association for probiotics and prebiotics consensus statement on the scope and appropriate use of the term probiotic. Nat Rev Gastroenterol Hepatol. 2014; 11(8):506-14.

11. Sanders ME, Marco ML. Food formats for effective delivery of probiotics. Annu Rev Food Sci Technol. 2010; 1:65-85.

12. Vinderola G, Reinheimer J, Salminen S. The enumeration of probiotic issues: From unavailable standardised culture media to a recommended procedure? Int Dairy J. 2019; 96:58-65.

13. Binda S, Hill C, Johansen E, Obis D, et al. Criteria to Qualify Microorganisms as "Probiotic" in Foods and Dietary Supplements. Front Microbiol. 2020; 11:1662.

14. Villena JC, Salva MS, Nuñez MS, CorzoJ, et al. Probiotics for Everyone! The Novel Immunobiotic Lactobacillus rhamnosus CRL1505 and the Beginning of Social Probiotic Programs in Argentina. Int J Biotechnol Wellness Ind. 2012; 1(3):189-98.

15. Pace F, Pace M, Quartarone G. Probiotics in digestive diseases: Focus on Lactobacillus GG. Minerva Gastroenterol Dietol. 2015; 61(4):273-92.

16. Dronkers TMG, Ouwehand AC, Rijkers GT.Global analysis of clinical trials with probiotics. Heliyon. 2020; 6(7):e04467.

17. Majamaa H, Isolauri E, Saxelin M, Vesikari T. Lactic acid bacteria in the treatment of acute rotavirus gastroenteritis. J Pediatr Gastroenterol Nutr. 1995; 20(3):333-8.

18. Wieërs G, Belkhir L, Enaud R, Leclercq S, et al. How Probiotics Affect the Microbiota. Front Cell Infect Microbiol. 2020; 9:454.

19. Sanders ME, Akkermans LMA, Haller D, Hammerman C, et al. Safety assessment of probiotics for human use. Gut Microbes. 2010; 1(3):164-85.

20. EFSA Panel on Biological Hazards (BIOHAZ), Ricci A, Allende A, Bolton D, et al. Scientific Opinion on the update of the list of QPS $\square$ recommended biological agents intentionally added to food or feed as notified to EFSAt. EFSA J. 2017; 15(3):e04664.

21. Bernardeau M, Guguen M, Vernoux JP. Beneficial lactobacilli in food and feed: Long-term use, biodiversity and proposals for specific and realistic safety assessments. FEMS Microbiol Rev. 2006; 30(4):487-513.

22. Bernardeau M, Vernoux JP, Henri-DubernetS, Guéguen M. Safety assessment of dairy microorganisms: the Lactobacillus genus. Int J Food Microbiol. 2008; 126(3):278-85.

23. Hojsak I, Fabiano V, Pop TL, Goulet O, et al. Guidance on the use of probiotics in clinical practice in children with selected clinical conditions and in specific vulnerable groups. Acta Paediatr. 2018; 107(6):927-37.

24. Hempel S, Newberry S, Ruelaz A, Wang Z, et al. Safety of probiotics used to reduce risk and prevent or treat disease. Evid Rep Technol Assess (Full Rep). 2011; (200):1-645.
25. Tynkkynen S, Singh K V, Varmanen P. Vancomycin resistance factor of Lactobacillus rhamnosus GG in relation to enterococcal vancomycin resistance (van) genes. Int J Food Microbiol. 1998; 41(3):195-204.

26. Martín-Muñoz MF, Fortuni M, Caminoa M, Belver T, et al. Anaphylactic reaction to probiotics: Cow's milk and hen's egg allergens in probiotic compounds. Pediatr Allergy Immunol. 2012; 23(8):778-84.

27. Braegger C, Chmielewska A, Decsi T, Kolacek S, et al. Supplementation of infant formula with probiotics and/ or prebiotics: A systematic review and comment by the ESPGHAN committee on nutrition. J Pediatr Gastroenterol Nutr. 2011; 52(2):238-50.

28. Choi S, Hwang YJ, Shin MJ, Yi H. Difference in the gut microbiome between ovariectomy-induced obesity and diet-induced obesity. J Microbiol Biotechnol. 2017; 27(12):2228-36.

29. Mennini M, Dahdah L, Artesani MC, Fiocchi A, Martelli A. Probiotics in asthma and allergy prevention. Front Pediatr. 2017; 5:165.

30. Guarner F, Requena T, Marcos A. Consensus statements from the workshop "Probiotics and health: Scientific evidence." Nutr Hosp. 2010; 25(5):700-4.

31. Schnadower D, Tarr PI, Casper TC, Gorelick MH, et al. Lactobacillus rhamnosus GG versus Placebo for Acute Gastroenteritis in Children. N Engl J Med. 2018; 379(21):2002-14.

32. Szajewska H, Canani RB, Guarino A, Hojsak I, et al. Probiotics for the prevention of antibiotic-associated diarrhea in children. J Pediatr Gastroenterol Nutr. 2016; 62(3):495-506.

33. Anabrees J,IndrioF, Paes B, AlFaleh K. Probiotics for infantile colic: A systematic review. BMC Pediatr. 2013; 13:186.

34. Kalliomäki M, Salminen S, Arvilommi H, Kero P, et al. Probiotics in primary prevention of atopic disease: A randomised placebo-controlled trial. Lancet. 2001; 357(9262):1076-9.

35. Dinleyici EC, Eren M, Ozen M, Yargic ZA, Vandenplas Y. Effectiveness and safety of Saccharomyces boulardii for acute infectious diarrhea. Expert Opin Biol Ther. 2012; 12(4):395410.

36. Szajewska H, Wanke M, Patro B. Meta-analysis: The effects of Lactobacillus rhamnosus GG supplementation for the prevention of healthcare-associated diarrhoea in children. Aliment Pharmacol Ther. 2011; 34(9):1079-87.

37. Sung V, D'Amico F, Cabana MD, Chau K, et al. Lactobacillus reuteri to treat infant colic: a meta-analysis. Pediatrics. 2018; 141(1):e20171811.

38. Savino F, Montanari P, Galliano I, Daprà V, Bergallo M. Lactobacillus rhamnosus GG (ATCC 53103) for the management of infantile colic: a randomized controlled trial. Nutrients. 2020; 12(6):1693.

39. Torres S, Fabersani E, Marquez A, Gauffin-Cano P. Adipose tissueinflammation and metabolic syndrome. The proactive role of probiotics. Eur J Nutr. 2019; 58(1):27-43.

40. Ettinger G, MacDonald K, Reid G, Burton JP. The influence of the human microbiome and probiotics on cardiovascular health. Gut Microbes. 2014; 5(6):719-28.

41. Daley D. The evolution of the hygiene hypothesis: The role of early-life exposures to viruses and microbes and their relationship to asthma and allergic diseases. Curr Opin Allergy Clin Immunol. 2014; 14(5):390-6.

42. Walker WA, Iyengar RS. Breast milk, microbiota, and intestinal immune homeostasis. Pediatr Res. 2015; 77(12):220-8.

43. Kim KS, Hong SW, Han D, Yi J, et al. Dietary antigens limit mucosal immunity by inducing regulatory $\mathrm{T}$ cells in the small intestine. Science. 2016; 351(6275):858-63. 
44. Abrahamsson TR, Jakobsson HE, Andersson AF, Björkstén $B$, et al. Low diversity of the gut microbiota in infants with atopic eczema. J Allergy Clin Immunol. 2012; 129(2):434-40. e1-2.

45. Suzuki K, Kawamoto S, Maruya M, Fagarasan S. GALT: Organization and dynamics leading to IgA synthesis. Adv Immunol. 2010; 107:152-85.

46. Berni Canani R, Di Costanzo M, Bedogni G, Amoroso A, et al. Extensively hydrolyzed casein formula containing Lactobacillus rhamnosus GG reduces the occurrence of other allergic manifestations in children with cow's milk allergy: 3-year randomized controlled trial. J Allergy Clin Immunol. 2017; 139(6):1906-13.e4.

47. Rautava S, Kalliomäki M, Isolauri E. Probiotics during pregnancy and breast-feeding might confer immunomodulatory protection against atopic disease in the infant. J Allergy Clin Immunol. 2002; 109(1):119-21.

48. Cuello-García CA, Brozek JL, Fiocchi A, Pawankar R, et al. Probiotics for the prevention of allergy: A systematic review and meta-analysis of randomized controlled trials. J Allergy Clin Immunol. 2015; 136(4):952-61.

49. Mesa MD, Loureiro B, Iglesia I, Fernández González S, etal. The evolving microbiome from pregnancy to early infancy: a comprehensive review. Nutrients. 2020; 12(1):133.

50. Diggikar S. Neonatal microbiome: A complex, invisible organ and its evolving role in neonatal illness and beyond. J Clin Neonatol. 2019; 8(1):5-9.

51. Dermyshi E, Wang Y, Yan C, Hong W, et al. The “Golden Age" of Probiotics: a systematic review and meta-analysis of randomized and observational studies in preterminfants. Neonatology. 2017; 112(1):9-23.

52. Athalye-Jape G, Deshpande G, Rao S, Patole S. Benefits of probiotics on enteral nutrition in preterm neonates: A systematic review. Am J Clin Nutr. 2014; 100(6):1508-19.

53. Kane AF, Bhatia AD, Denning PW, Shane AL, Patel RM. Routine Supplementation of Lactobacillus rhamnosus GG and Risk of Necrotizing Enterocolitis in Very Low Birth Weight Infants. J Pediatr. 2018; 195:73-9.e2.

54. van den Akker CHP, van Goudoever JB, Shamir R, Domellöf $\mathrm{M}$, et al. Probiotics and Preterm Infants: A Position Paper by the European Society for Paediatric Gastroenterology Hepatology and Nutrition Committee on Nutrition and the European Society for Paediatric Gastroenterology Hepatology and Nutrition Working Group for Pr. J Pediatr Gastroenterol Nutr. 2020; 70(5):664-80.

55. Su GL, Ko CW, Bercik P, Falck-Ytter Y, et al. AGA Clinical Practice Guidelines on the role of probiotics in the management of gastrointestinal disorders. Gastroenterology. 2020; 159(2):697-705.

56. Plaza-Diaz J, Ruiz-Ojeda FJ, Gil-Campos M, Gil A. Mechanisms of Action of Probiotics. Adv Nutr. 2019; 10(Suppl 1):S49-66.

57. Gu Q, Li P. Biosynthesis of Vitamins by Probiotic Bacteria. In: Rao V, Rao L (eds). Probiotics and Prebiotics in Human Nutrition and Health. Rijeka: IntechOpen; 2016.Págs.13548.

58. Aziz N, Bonavida B. Activation of natural killer cells by probiotics. For Immunopathol Dis Therap. 2016; 7(1-2):41-55.

59. Azad MAK, Sarker M, Li T, Yin J. Probiotic Species in the Modulation of Gut Microbiota: An Overview. Biomed Res Int. 2018; 2018:9478630.

60. Ohland CL, MacNaughton WK. Probiotic bacteria and intestinal epithelial barrier function. Am J Physiol Gastrointest Liver Physiol. 2010; 298(6):G807-19.

61. Capurso L. Thirty Years of Lactobacillus rhamnosus GG A Review. J Clin Gastroenterol. 2019; 53 (Suppl 1):S1-41. 